\title{
The Simulink of Social Communication Networks Based on An Improved Scale-free Network Model
}

\author{
Zhengyifan Chen \\ School of North China Electric Power University, Baoding 071000, China
}

519604267@qq.com

Keywords: Scale-free network model, the evolution of social communication networks

\begin{abstract}
Information is spread quickly in today's tech-connected communication network and we want to know more about the communication networks' relationships. In this paper, an improved scale-free network model of social communication is introduced to simulate the evolution of social communication networks. In this model, we regard the subjective factors of participants and development of social network as the main causes of network evolution. The two kind of participants (active participants and common participants) divided by the desire to communicate with others, meanwhile the desire to communicate changes with the development of economic and education are taken into consideration. Finally, we get a picture of the evolution of social communication networks from 1870 to 2010. The algorithm can provide guidance for the study of social communication networks.
\end{abstract}

\section{Introduction}

A History of Communications is roughly divided into 5 periods: the age of speech, the age of manuscripts, the age of print, the age of audiovisual media and the age of the internet ${ }^{[2]}$. With the development of science and technology, social information system towards stronger. In social network, any two participants are linked to each other if there is an interaction between them, through which new information can diffuse from one to another participant, eventually influence the whole network. So we try to simulate the evolution of social communication networks by using an improved scale-free network model, and the method can be used to study the flow of information in social communication networks

\section{Scale-free Network Model ${ }^{[2]}$}

- Edge: Edge is the ditch that two points interact with each other. Focused on the present network that regard participants as points, the two most direct connections are to communicate or disseminate information.

- Node: The nodes in the network model refer to the participants in the social communication network. It should be noted that those nodes not only represent single person, but also the Influential media organizations and information exchange platforms in the communication network.

- Neighbor: the most close nodes to the target node.

- Second-degree node: the neighbors of the target node's neighbor.

- Degree of node: the number of edges connected with the target node directly. Assume that network $G=(V, E)$ is consists of $|V|=N$ nodes and $|E|=M$ sides. Node degree can be expressed as:

$$
k_{i}=\sum_{j \in G, j \neq i} \delta_{i j}
$$

If node $\mathrm{i}$ and node $\mathrm{j}$ is not connected, $\delta_{i j}=0$. If node $\mathrm{i}$ and node $\mathrm{j}$ is connected $\delta_{i j}=1$.

- Rewiring probability $p$ : in a period, $m$ edges in the network are broken, at meanwhile, $m$ edges are rebuilt between nodes with probability $p$. Each code has probability p to generate a new edge. 
- Diameter, Radius, and Centrality: The longest path between any two nodes in a graph $G$ is called the diameter of G, denoted diameter. Let the longest path from a node $u$ to all other nodes of a connected graph be defined as the radius of node $\mathrm{u}$, denoted radius. Then the node with the smallest radius is the center of the graph, and the largest radius over all nodes, is the graph's diameter. The diameter of a graph is also equal to the length of the longest directed path in G.

- Average path length $d$ : Compute the direct path between all pairs of nodes, and then average them. The answer is $d$, average path length of a network.

- Active nodes: the nodes always try to connect with any other nodes, not only its neighbors and second-degree nodes. Unfortunately, there is an upper limit $d_{\max }$ of its degree. And $d_{\max }$ is capable of being influenced by Level of technology and communication network changes. When the degree of an active node reached the upper limit, it will abandon one of its neighboring nodes whose degree is smallest. $Y_{x}(t)$, whose value will mutate in different period, is a function to describe the active nodes' ability to communicate with other nodes. The number of active nodes in the network is shown as $n_{i}$ 。

$$
n_{i}=\frac{N_{i}}{N} \times 100 \%
$$

Where $N_{i}$ is the number of active nodes, while $N$ is the number of total nodes in in the social network. The value of $n_{i}$ is increasing with time, and at the same time the first derivative of $n_{i}$ with respect to time also grows during different development stages. It is easily to understand that more and more people have stronger desire to communicate with others if they have much time and money. We assume that:

$$
d_{\max }(t)=k \times Y_{x}(t)
$$

Common nodes: the nodes in the network except the active nodes. There are two communicating rules for common codes, which are listed as follows:

- Try to communicate with the codes who is closer to itself geographically as well as has greater degree.

- Try to communicate with the neighbors' second-degree nodes. For example, people establish connect with his neighbor's distant relatives. Neighbors are close in geography and there are direct connections between neighbor and his relatives. There is also an upper limit of common nodes' degree $d_{\text {avg }}(t)$, which has changed over time.(share the same description function with active nodes )

\section{Result \&Analysis}

In order to make such a network alive, the network is reconnected in each period. Aimed at controlling the speed of network evolution, we set up a connect probability and the social network will be reconnected with the probability. Based on the, I simulate the change of a social network from 1870 to 2010 which still has 100 nodes by MATLAB. The result is shown as Figure 1. 


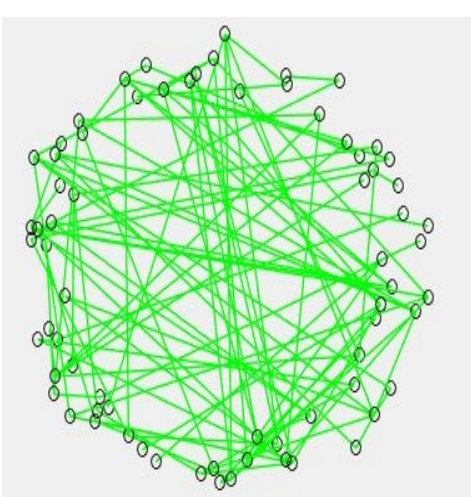

1960

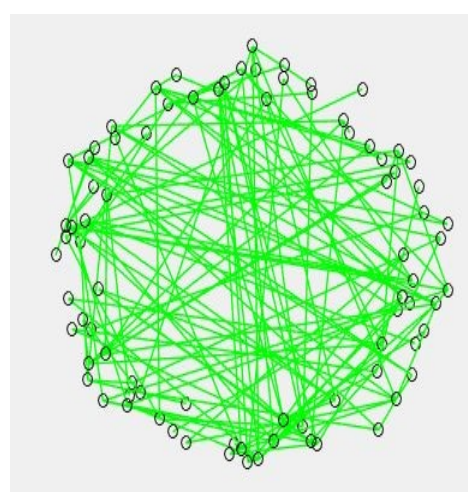

2010

Figure 1 the evolution process of social communication network based on our rules Through the comparison of Figure 7, the average path length is chosen out to measure the speed of information diffusion. The results that we simulate the changes of average path length over time are shown as Figure 2

$1870-2010$

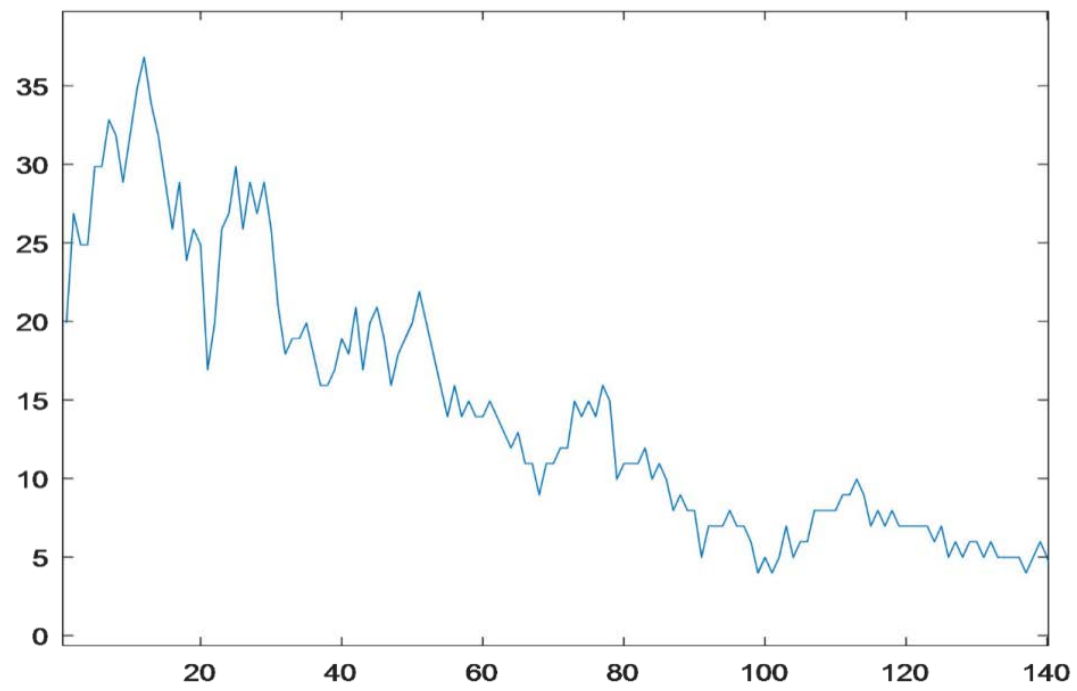

Figure 2 average path length (Y-axis) changes with year (X-axis)

\section{Summary}

We find that the network's evolution rules under new situation is more complex. Driven by the subjective desire, there are greater degree and shorter average path length of nodes in the network. All those are in good agreement with the actual situation. So, our simulation models can be used in engineering practice.

\section{References}

[1] Ted G. Lewis. NETWORK SCIENCE: Theory and Applications [M]. New York: A JOHN WILEY \& SONS, INC, 2009

[2] Marshall T. Poe. A History of Communications: Media and Society from the Evolution of Speech to the Internet [M]. New York: Cambridge University Press, 2010 